\title{
THE HAUSDORFF-YOUNG INEQUALITY FOR ORLICZ SPACES ON COMPACT HYPERGROUPS
}

\author{
VISHVESH KUMAR AND RITUMONI SARMA
}

\begin{abstract}
We prove the classical Hausdorff-Young inequality for the Lebesgue spaces on a compact hypergroup using interpolation of sublinear operators. We use this result to prove the Hausdorff-Young inequality for Orlicz spaces on a compact hypergroup.
\end{abstract}

\section{INTRODUCTION}

For a locally compact abelian group $G, 1 \leq p \leq 2$ and $p^{\prime}=\frac{p}{p-1}$, the classical Hausdorff-Young inequality says, "If $f \in L^{1}(G) \cap L^{p}(G)$ then the Fourier transform $\widehat{f} \in L^{p^{\prime}}(\widehat{G})$ and $\|\widehat{f}\|_{p^{\prime}} \leq\|f\|_{p .}$.

This is proved by using Riesz convexity complex interpolation theorem between $p=1$ and $p=2$ (the Plancherel theorem).

Hypergroups are probabilistic generalization of locally compact groups where the convolution of two points is a point mass measure. In the spirit of locally compact abelian group, Vrem [15] and Degenfeld-Schonburg [3] proved Hausdorff-Young inequality for compact hypergroups and commutative hypergroups respectively using the complex interpolation technique.

It is well known that between any two Lebesgue spaces there is an Orlicz space which is not a Lebesgue space. M. M. Rao [8] studied the HausdorffYoung inequality for Orlicz spaces on locally compact abelian groups. In fact, the celebrated work of M. M. Rao in the context of Orlicz spaces on locally compact groups (see $[8,9,12,10]$ ) motivates the authors for studying Orlicz spaces of hypergroups [7]. In this article, we study the classical Hausdorff-Young inequality with an enlargement of the space, namely, an Orlicz space on a compact hypergroup. This work can be considered as a generalization of [8] as well as [15].

It is to be noted that the Riesz convexity theorem is useful to the $L^{p_{-}}$ spaces only. For Orlicz spaces, results are obtained first by extending a key inequality of Hausdorff-Young in the form of Hardy-Littlewood [5, pg. 170].

2010 Mathematics Subject Classification. Primary 43A62, 46E30; Secondary 43A30.

Key words and phrases. Compact hypergroups, Orlicz spaces, Hausdorff-Young inequality, classical Hardy-Littlewood inequality, Fourier transform. 
It is worth mentioning here that we apply the method of Hausdorff-HardyLittlewood [5] which does not require the Plancherel theorem.

In Section 2, we present needful basics of Orlicz spaces and compact hypergroups in the form we use in the sequel. In Section 3, we prove the classical Housdorff-Young inequality for compact hypergroups; this proof is slightly different from that of Vrem [15]. Then we prove a key Lemma which occupies a major part of this section, and finally, we prove the HausdorffYoung inequality for Orlicz spaces on compact hypergroups.

\section{PRELIMINARIES}

2.1. Basics of Orlics spaces. For basics of Orlicz spaces one can refer to two excellent monographs by Rao and Ren [11, 12] and articles [8, 9, 10, 7]. However we present a few definitions and results here in the form we need.

A non-zero convex function $\Phi: \mathbb{R} \rightarrow[0, \infty]$ is called a Young function if it is an even function with $\Phi(0)=0$. For any Young function $\Phi$, and $y \in \mathbb{R}$, the complementary function $\Psi$ of $\Phi$ is given by ,

$$
\Psi(y)=\sup \{x|y|-\Phi(x): x \geq 0\}
$$

which is also a Young function. If $\Psi$ is the complementary function of $\Phi$ then $\Phi$ is the complementary function of $\Psi$; the pair $(\Psi, \Phi)$ is called a complementary pair. In fact, a complementary pair of Young functions satisfies

$$
x y \leq \Phi(x)+\Psi(y) \quad(x, y \geq 0) .
$$

If a complementary pair of Young functions $(\Phi, \Psi)$ satisfies $\Phi(1)+\Psi(1)=1$ then the pair $(\Phi, \Psi)$ called a normalized complementary pair.

Let $K$ be a compact hypergroup with a left Haar measure $m$. Denote the set of all complex valued $m$-measurable functions on $K$ by $L^{0}(K)$. Given a Young function $\Phi$, the modular function $\rho_{\Phi}: L^{0}(K) \rightarrow \mathbb{R}$ is defined by $\rho_{\Phi}(f):=\int_{K} \Phi(|f|) d m$ and the Orlicz space is defined by

$$
L^{\Phi}(K):=\left\{f \in L^{0}(K): \rho_{\Phi}(a f)<\infty \text { for some } a>0\right\} .
$$

Then the Orlicz space is a Banach space with respect to the norm $N_{\Phi}(\cdot)$ defined by

$$
N_{\Phi}(f):=\inf \left\{r>0: \int_{K} \Phi\left(\frac{|f|}{r}\right) d m \leq \Phi(1)\right\} .
$$

Let $C_{c}(K)$ denote the space of complex valued continuous functions with compact support on $K$. The closure of $C_{c}(K)$ inside $L^{\Phi}(K)$ is denoted by $M^{\Phi}(K)$. Another useful description of $M^{\Phi}(K)$ is that $f \in M^{\Phi}(K)$ if and only if for every $\alpha>0, \alpha f \in L^{\Phi}(K)$. 
An operator $T: X \rightarrow Y$, where $X$ is a Banach space and $Y$ is a Banach lattice, is called sublinear if $|T(\alpha f)|=|\alpha||T(f)|$ for $\alpha \in \mathbb{C}, f \in X$ and $\left|T\left(f_{1}+f_{2}\right)\right| \leq\left|T\left(f_{1}\right)\right|+\left|T\left(f_{2}\right)\right|$ for $f_{1}, f_{2} \in X$ (see [12, p. 193] and [2]).

The partial order $\prec$ on th set of all Young functions is defined as: $\Phi_{1} \prec \Phi_{2}$ whenever $\Phi_{1}(a x) \leq b \Phi_{2}(x)$ for $|x| \geq x_{0}>0$ and $\Phi_{2}(c x) \leq d \Phi_{1}(x)$ for all $|x| \leq x_{1}$, where $a, b, c, d, x_{0}$ and $x_{1}$ are fixed positive constants independent of $x$. In particular, for $L^{p}$-spaces $p \geq 1$ we can see that $a=b=c=$ $d=1, x_{1} \geq 1$ and $x_{0} \geq 1$. With the help of this ordering we can define inclusion relation in Orlicz spaces: if $\Phi_{1}, \Phi_{2}$ are continuous Young functions and $\Phi_{1} \prec \Phi_{2}$ then $L^{\Phi_{2}}(K) \subset L^{\Phi_{1}}(K)$ and $N_{\Phi_{1}}(\cdot) \leq \alpha N_{\Phi_{2}}(\cdot)$ for some $\alpha>0$. If $K$ is a discrete space then $L^{\Phi}(K)$ becomes $\ell^{\Phi}(K)$ and in this case $\Phi_{1} \prec \Phi_{2}$ implies that $\ell^{\Phi_{1}} \subset \ell^{\Phi_{2}}$ and $N_{\Phi_{2}}(\cdot) \leq \beta N_{\Phi_{1}}(\cdot)$ for some $\beta>0$. The following result is well-known (see [12, Lemma 1, p. 209]).

Lemma 2.1. Let $\left(\Phi_{i}, \Psi_{i}\right), i=1,2$ be complementary pairs of continuous Young functions and $\Phi_{1} \prec \Phi_{2}$. Then $\Psi_{2} \prec \Psi_{1}$.

2.2. Basics of compact hypergroups. For the basics of compact hypergroups one can refer to standard books, monographs and research paper $[4,6,1,13,14,15,16]$. However we mention here certain results we need.

Let $K$ be a compact hypergroup with the normalized Haar measure $m$ and let $\widehat{K}=\left\{\pi^{\alpha}\right\}_{\alpha \in \Lambda}$, where $\Lambda$ is an index set, be the set of irreducible inequivalent continuous representations of $K$. The set $\widehat{K}$ equipped with the discrete topology is called the dual space of $K$. Vrem [16] showed that every irreducible representation $\left(\pi^{\alpha}, \mathcal{H}_{\pi^{\alpha}}\right)$ of a compact hypergroup is finite dimensional. For any $\pi^{\alpha} \in \widehat{K}$, the map $x \mapsto\left\langle\pi^{\alpha}(x) u, v\right\rangle$ for $u, v \in \mathcal{H}_{\pi^{\alpha}}$ is called a matrix coefficient function and is denoted by $\pi_{u, v}^{\alpha}$. Let $\pi^{\alpha}(x)=$ $\left[\pi_{i, j}^{\alpha}\right]_{\pi_{\pi^{\alpha} \times d_{\pi^{\alpha}}}}$ be the matrix representation of any $\left(\pi^{\alpha}, \mathcal{H}_{\pi^{\alpha}}\right)$ of dimension $d_{\pi^{\alpha}}$ with respect to an orthonormal basis $\left\{e_{i}\right\}_{i=1}^{d_{\pi^{\alpha}}}$ of $\mathcal{H}_{\pi^{\alpha}}$. For each pair $\pi^{\alpha}, \pi^{\beta} \in \widehat{K}$ there exists a constant $k_{\pi^{\alpha}} \geq d_{\pi^{\alpha}}$ such that

$$
\int_{K} \pi_{i, j}^{\alpha}(x) \overline{\pi_{k, l}^{\beta}(x)} d m(x)= \begin{cases}\frac{1}{k_{\pi^{\alpha}}} & \text { when } i=k, j=l, \text { and } \alpha=\beta \\ 0 & \text { otherwise. }\end{cases}
$$

If $K$ is a compact group then $k_{\pi^{\alpha}}=d_{\pi^{\alpha}}$ [16, Theorem 2.6].

For each $\pi^{\alpha} \in \widehat{K}$, the Fourier transform $\widehat{f}$ of $f \in L^{1}(K)$ is defined as $\widehat{f}\left(\pi^{\alpha}\right)=\left[\left\langle f, \pi_{i, j}^{\alpha}\right\rangle\right]_{i, j=1}^{d_{\pi^{\alpha}}}$. where $\langle\cdot, \cdot\rangle$ is the inner product on $L^{2}(K)$. Since $L^{\Phi}(K) \subset L^{1}(K)$, the Fourier transform of $f \in L^{\Phi}(K)$ is well defined. For $f \in L^{2}(K)$, we have

$$
f=\sum_{\pi^{\alpha} \in \widehat{K}} k_{\pi^{\alpha}} \sum_{i, j=1}^{d_{\pi^{\alpha}}} \widehat{f}\left(\pi^{\alpha}\right)_{i, j} \pi_{i, j}^{\alpha}
$$


and the series converges in $L^{2}(K)$ [16, Corollary 2.10]. Hence,

$$
\|f\|_{2}^{2}=\sum_{\pi^{\alpha} \in \widehat{K}} k_{\pi^{\alpha}} \sum_{i, j=1}^{d_{\pi^{\alpha}}}\left|\widehat{f}\left(\pi^{\alpha}\right)_{i, j}\right|^{2}=\sum_{\pi^{\alpha} \in \widehat{K}} k_{\pi^{\alpha}}\left\|\widehat{f}\left(\pi^{\alpha}\right)\right\|_{2}^{2}
$$

\section{The MAIN RESUlT}

Throughout this section, we assume that $K$ is a compact hypergroup and $\widehat{K}=\left\{\pi^{\alpha}\right\}_{\alpha \in \Lambda}$, where $\Lambda$ is an index set, be the set of irreducible inequivalent continuous representations of $K$. At times, we also use an element of $\Lambda$ to describe an element of $\widehat{K}$. From now onwards, we assume that the pair of complementary continuous Young functions $(\Phi, \Psi)$ is a normalized pair. Note that continuity of a Young function guaranties the existence of its derivative [11, Corollary 2, p. 10].

For $f \in L^{\Phi}(K)$ define $F_{f}: \Lambda \rightarrow \mathbb{R}_{+}$by

$$
F_{f}^{2}(\alpha)=\sum_{i, j=1}^{d_{\pi^{\alpha}}} \frac{\left|\widehat{f}\left(\pi^{\alpha}\right)_{i, j}\right|^{2}}{k_{\pi^{\alpha}}}=\frac{\operatorname{Tr}\left(\widehat{f}\left(\pi^{\alpha}\right)^{*} \widehat{f}\left(\pi^{\alpha}\right)\right)}{k_{\pi^{\alpha}}} \text { for } \alpha \in \Lambda,
$$

where for a matrix $A, A^{*}=\bar{A}^{t}$. Now, the gauge norm of $f$ and $F_{f}$ are defined as follows:

$$
N_{\Phi}(f):=\inf \left\{r>0: \int_{K} \Phi\left(\frac{|f|}{r}\right) d m(x) \leq \Phi(1)\right\},
$$

and

$$
N_{\Phi}\left(F_{f}\right):=\inf \left\{r>0: \sum_{\alpha \in \Lambda} \Phi\left(\frac{F_{f}(\alpha)}{r}\right) k_{\pi^{\alpha}}^{2} \leq \Phi(1)\right\} .
$$

The space $\left(L^{\Phi}(K), N_{\Phi}(\cdot)\right)$ and $\left(\ell^{\Phi}(\widehat{K}), N_{\Phi}(\cdot)\right)$ are Orlicz spaces. If $\Phi$ is continuous then there exists $k_{0}:=N_{\Phi}\left(F_{f}\right)$ such that inequality in (3.3) is an equality with $r=k_{0}$, i.e., $\sum_{\alpha \in \Lambda} \Phi\left(\frac{F_{f}(\alpha)}{k_{0}}\right) k_{\pi^{\alpha}}^{2}=\Phi(1)$.

The following theorem presents a proof of Hausdorff-Young inequality moderately different from Vrem's proof [15, Theorem 3.10].

Theorem 3.1. Let $K$ be a compact hypergroup with the normalized Haar measure $m$ and let $f \in L^{p}(K, m)$ for $1 \leq p \leq 2$. Suppose $\frac{1}{p}+\frac{1}{p^{\prime}}=1$. If $\left\{F_{f}(\alpha): \alpha \in \Lambda\right\}$ is as in (3.1) then

$$
\|\hat{f}\|_{p^{\prime}}=\left(\sum_{\alpha \in \Lambda} F_{f}(\alpha)^{p^{\prime}} k_{\pi^{\alpha}}^{2}\right)^{\frac{1}{p^{\prime}}} \leq\|f\|_{p} .
$$

Proof. Let $\ell^{p^{\prime}}\left(k^{2}\right)$ denote the space of $p^{\prime}$ th summable sequences on $\widehat{K}$ relative to the weights $\left\{k_{\pi^{\alpha}}^{2}: \alpha \in \widehat{K}\right\}$. Define the operator $T: L^{p}(K) \rightarrow \ell^{p^{\prime}}\left(k^{2}\right)$ by 
$T(f)=F_{f}$, where $F_{f}$ is given by (3.1). It is easy to see that $T$ is sub-linear. Moreover, by Parseval formula,

$$
\|T f\|_{2}^{2}=\sum_{\alpha \in \Lambda} F_{f}(\alpha)^{2} k_{\pi^{\alpha}}^{2}=\sum_{\alpha \in \Lambda} \sum_{i, j=1}^{d_{\pi^{\alpha}}}\left|\hat{f}\left(\pi_{i, j}^{\alpha}\right)\right|^{2} k_{\pi^{\alpha}}=\|f\|_{2}^{2} .
$$

By definition,

$$
\|T f\|_{p^{\prime}}= \begin{cases}\left(\sum_{\alpha \in \Lambda} F_{f}(\alpha)^{p^{\prime}} k_{\pi^{\alpha}}^{2}\right)^{\frac{1}{p^{\prime}}} & 1 \leq p^{\prime}<\infty \\ \sup _{\alpha \in \Lambda} \frac{F_{f}(\alpha)}{k_{\pi^{\alpha}}} & p^{\prime}=\infty\end{cases}
$$

Now,

$$
\begin{aligned}
F_{f}(\alpha)=\left(\frac{1}{k_{\pi^{\alpha}}} \sum_{i, j=1}^{d_{\pi^{\alpha}}}\left|\widehat{f}\left(\pi^{\alpha}\right)_{i, j}\right|^{2}\right)^{\frac{1}{2}} & \leq \frac{1}{k_{\pi^{\alpha}}^{\frac{1}{2}}} \sum_{i, j=1}^{d_{\pi^{\alpha}}}\left|\widehat{f}\left(\pi^{\alpha}\right)_{i, j}\right| \\
& \leq \frac{1}{k_{\pi^{\alpha}}^{\frac{1}{2}}} \int_{K}|f(x)| \sum_{i, j=1}^{d_{\pi^{\alpha}}}\left|\pi^{\alpha}(x)_{i, j}\right| d m(x) .
\end{aligned}
$$

Using Cauchy-Schwarz inequality, we get

$$
F_{f}(\alpha) \leq \frac{1}{k_{\pi^{\alpha}}^{\frac{1}{2}}} \int_{K}|f(x)|\left(\sum_{i, j=1}^{d_{\pi^{\alpha}}}\left|\pi^{\alpha}(x)_{i, j}\right|^{2}\right)^{\frac{1}{2}} d_{\pi^{\alpha}} d m(x) .
$$

Since $\pi^{\alpha}(x)$ is a $d_{\pi^{\alpha}} \times d_{\pi^{\alpha}}$ orthogonal matrix and the hyperdimension $k_{\pi^{\alpha}} \geq$ $d_{\pi}^{\alpha}$, we get

$$
F_{f}(\alpha) \leq\left(\frac{d_{\pi^{\alpha}}}{k_{\pi^{\alpha}}}\right)^{\frac{1}{2}} \int_{K}|f(x)| d_{\pi^{\alpha}} d m(x) \leq k_{\pi^{\alpha}} \int_{K}|f(x)| d m(x) .
$$

Hence, we have

$$
\|T f\|_{\infty} \leq\|f\|_{1}
$$

From (3.5) and (3.6), the required inequality follows by the Riesz-Thorin theorem for sub-linear operators [2].

The proof of our main result depends on the following key lemma which is an extension of an important inequality in the case of $L^{p}$ due to Hardy and Littlewood [5].

Lemma 3.2. Let $K$ be a compact hypergroup with the normalized Haar measure $m$ and let $(\Phi, \Psi)$ be a pair of continuous normalized Young functions such that

(i) $\Phi \prec \Phi_{0}$, where $\Phi_{0}(t)=\frac{1}{2}|t|^{2}$,

(ii) $\Psi^{\prime}(t) \leq c_{0} t^{p}, \forall t \geq 0$, for some $p \geq 1$, and for some $c_{0}>0$. 
Suppose $\Lambda_{0}$ is a finite subset of $\Lambda$. Define $f_{\Lambda_{0}}: K \rightarrow \mathbb{C}$ by

$$
f_{\Lambda_{0}}(x):=\sum_{\alpha \in \Lambda_{0}} k_{\pi^{\alpha}} \sum_{i, j=1}^{d_{\pi^{\alpha}}} c_{i, j}^{\alpha} \pi_{i, j}^{\alpha}(x),
$$

where $c_{i, j}^{\alpha} \in \mathbb{C}$. If $F_{\Lambda_{0}}=F_{f}$ is as in (3.1) with $f=f_{\Lambda_{0}}$, then

$$
N_{\Psi}\left(F_{\Lambda_{0}}\right) \leq \bar{r}_{0} N_{\Phi}\left(f_{\Lambda_{0}}\right),
$$

where $\overline{r_{0}}>0$ depends only on $\Phi$ and the ordering $\prec$.

Proof. Let $\Lambda_{0}$ be a finite subset of $\Lambda$. If $f_{\Lambda_{0}}$ is as in the statement of the lemma, $\hat{f}_{\Lambda_{0}}\left(\pi^{\alpha}\right)_{i, j}=c_{i, j}^{\alpha} \chi_{\Lambda_{0}}(\alpha)$. For simplicity of expressions, we set $S_{\Phi}\left(f_{\Lambda_{0}}\right)=N_{\Phi}\left(F_{f_{\Lambda_{0}}}\right)$. For a non-zero $f \in L^{\Phi}(K)$, the Fourier coefficients $\hat{f}\left(\pi^{\alpha}\right)_{i, j}$ of $f$ are denoted by $\tilde{c}_{i, j}^{\alpha}$. Let $\tilde{f}_{\Lambda_{0}}$ be the function given by (3.7) with $c_{i, j}^{\alpha}=\tilde{c}_{i, j}^{\alpha}$. Following an idea of Hardy and Littlwood [5], we define,

$$
M=M_{\Phi}\left(\Lambda_{0}\right):=\sup \left\{\frac{S_{\Psi}\left(\tilde{f}_{\Lambda_{0}}\right)}{N_{\Phi}(f)}: f \neq 0\right\} .
$$

We prove the lemma in three steps.

STEP I. $M<\infty$.

Since $M$ is described by a ratio of norms, without loss of generality we assume that $S_{\Psi}\left(\tilde{f}_{\Lambda_{0}}\right)=1$ to find a bound on $M$. It follows by using continuity of $\Psi$ and the definition of the gauge norm (with $k_{0}=1$ ) that

$$
\sum_{\alpha \in \Lambda} \Psi\left(F_{\tilde{f}_{\Lambda_{0}}}(\alpha)\right) k_{\pi^{\alpha}}^{2}=\Psi(1) .
$$

Since $k_{\pi^{\alpha}} \geq 1, F_{\tilde{f}_{\Lambda_{0}}}(\alpha)=0$ for $\alpha \in \Lambda-\Lambda_{0}$ and $0<\Psi(1)<1$, at least one term on the left hand side of (3.10) is greater than or equal to $\frac{\Psi(1)}{\#\left(\Lambda_{0}\right)}$, where $\#\left(\Lambda_{0}\right)$ is the cardinality of $\Lambda_{0}$. If such a term is obtained for $\alpha=\alpha_{0} \in \Lambda_{0}$ then we have

$$
1 \leq \Psi^{-1}\left[\frac{\Psi(1)}{\#\left(\Lambda_{0}\right) k_{\pi^{\alpha}}^{2}}\right] \leq F_{\tilde{f}_{\Lambda_{0}}}\left(\alpha_{0}\right)
$$

Next,

$$
\begin{aligned}
F_{\tilde{f}_{\Lambda_{0}}}(\alpha)=\left(\frac{1}{k_{\pi^{\alpha}}} \sum_{i, j=1}^{d_{\pi^{\alpha}}}\left|\tilde{c}_{i, j}^{\alpha}\right|^{2}\right)^{\frac{1}{2}} & \leq \frac{1}{k_{\pi^{\alpha}}^{\frac{1}{2}}} \sum_{i, j=1}^{d_{\pi^{\alpha}}}\left|\tilde{c}_{i, j}^{\alpha}\right| \\
& \leq \frac{1}{k_{\pi^{\alpha}}^{\frac{1}{2}}} \int_{K}|f(x)| \sum_{i, j=1}^{d_{\pi^{\alpha}}}\left|\pi^{\alpha}(x)_{i, j}\right| d m(x) .
\end{aligned}
$$

Using Cauchy-Schwarz inequality, we get

$$
F_{\tilde{f}_{\Lambda_{0}}}(\alpha) \leq \frac{1}{k_{\pi^{\alpha}}^{\frac{1}{2}}} \int_{K}|f(x)|\left(\sum_{i, j=1}^{d_{\pi^{\alpha}}}\left|\pi^{\alpha}(x)_{i, j}\right|^{2}\right)^{\frac{1}{2}} d_{\pi^{\alpha}} d m(x) .
$$


Since $\pi^{\alpha}(x)$ is a $d_{\pi^{\alpha}} \times d_{\pi^{\alpha}}$ orthogonal matrix and the hyperdimension $k_{\pi^{\alpha}} \geq$ $d_{\pi}^{\alpha}$, we get

$$
F_{\tilde{f}_{\Lambda_{0}}}(\alpha) \leq\left(\frac{d_{\pi^{\alpha}}}{k_{\pi^{\alpha}}}\right)^{\frac{1}{2}} \int_{K}|f(x)| d_{\pi^{\alpha}} d m(x) \leq d_{\pi^{\alpha}} \int_{K}|f(x)| d m(x) .
$$

Now by Hölder's inequality

$$
F_{\tilde{f}_{\Lambda_{0}}}(\alpha) \leq d_{\pi^{\alpha}} N_{\Phi}(f) .
$$

Now by combining (3.11) and (3.13), we have

$$
\frac{1}{N_{\Phi}(f)} \leq\left(\frac{d_{\pi^{\alpha_{0}}}}{\Psi^{-1}\left(\frac{\Psi(1)}{\#\left(\Lambda_{0}\right) k_{\pi^{\alpha}}^{2}}\right)}\right)<\infty
$$

Since the right hand side of (3.14) is independent of $f$, we have $M<\infty$.

STEP II. $M$ is independent of $\Lambda_{0}$.

For $f_{\Lambda_{0}}$ as in (3.7), define $g$ by

$$
g(x):=\Psi^{\prime}\left(\frac{\left|f_{\Lambda_{0}}(x)\right|}{N_{\Psi\left(f_{\Lambda_{0}}\right)}}\right) \operatorname{sgn}\left(f_{\Lambda_{0}}(x)\right) .
$$

It is easy to see that $N_{\Phi}(g)=1$. It follows from [11, Proposition 9, p. 80] and [17, p. 175] that the Hölder's inequality is an equality, that is,

$$
N_{\Psi}\left(f_{\Lambda_{0}}\right)=N_{\Phi}(g) N_{\Psi}\left(f_{\Lambda_{0}}\right)=\int_{K} g(x) \bar{f}_{\Lambda_{0}}(x) d m(x) .
$$

Using the Parseval formula, we have

$$
\begin{aligned}
N_{\Psi}\left(f_{\Lambda_{0}}\right) & =\sum_{\alpha \in \Lambda_{0}} \sum_{i, j=1}^{d_{\pi^{\alpha}}} \hat{g}\left(\pi^{\alpha}\right)_{i, j} \overline{\hat{f}\left(\pi^{\alpha}\right)_{i, j}} \\
& \leq \sum_{\alpha \in \Lambda_{0}} k_{\pi^{\alpha}}^{2} F_{f_{\Lambda_{0}}}(\alpha) F_{\tilde{g}_{\Lambda_{0}}}(\alpha) \quad \text { (by Cauchy-Schwarz inequality) } \\
& \leq S_{\Phi}\left(f_{\Lambda_{0}}\right) S_{\Psi}\left(\tilde{g}_{\Lambda_{0}}\right) .
\end{aligned}
$$

By STEP I, we know that $S_{\Psi}\left(\tilde{g}_{\Lambda_{0}}\right) \leq M N_{\Phi}(g)=M$ and therefore

$$
\frac{N_{\Psi}\left(f_{\Lambda_{0}}\right)}{S_{\Phi}\left(f_{\Lambda_{0}}\right)} \leq S_{\Psi}\left(\tilde{g}_{\Lambda_{0}}\right) \leq M
$$

Note that $M \geq 1$. In fact, for $f=1$, we note that $N_{\Phi}(1)=1$ as the Haar measure $m$ is normalized. By continuity of $\Phi$,

$$
\sum_{\alpha \in \Lambda} \Phi\left(\frac{F_{\tilde{f}_{\Lambda_{0}}}(\alpha)}{S\left(\tilde{f}_{\Lambda_{0}}\right)}\right) k_{\pi^{\alpha}}^{2}=\Phi(1)
$$

Now, by choosing $\alpha_{0} \in \Lambda_{0}$ such that (3.11) holds, i.e., $F_{\tilde{f}_{\Lambda_{0}}}\left(\alpha_{0}\right)>1$, we have

$$
S_{\Psi}\left(\tilde{f}_{\Lambda_{0}}\right) \geq \frac{1}{\left[\Phi^{-1}\left(\frac{\Phi(1)}{k_{\pi^{\alpha_{0}}}^{2}}\right)\right]}=r_{0} \geq 1 \text { (say) }
$$


so that $M \geq \frac{S_{\Psi}\left(\tilde{f}_{0_{0}}\right)}{N_{\Phi}(f)} \geq r_{0} \geq 1$. By continuity of norms, there is a function $f_{\Lambda_{0}}$ such that $M=\frac{N_{\Psi}\left(f_{\Lambda_{0}}\right)}{S_{\Phi}\left(f_{\Lambda_{0}}\right)}$. Consequently, from (3.16) we get $S_{\Phi}\left(\tilde{g}_{\Lambda_{0}}\right)=M$. We fix this $f_{\Lambda_{0}}$ and set $g$ as in (3.15) for the remaining part of this step.

Suppose $S_{2}$ denotes $S_{\Psi}$ if $\Psi(x)=\frac{|x|^{2}}{2}$. Using the Bessel inequality, we get

$$
S_{2}^{2}\left(\tilde{g}_{\Lambda_{0}}\right)=\sum_{\alpha \in \Lambda_{0}} k_{\pi^{\alpha}}^{2} \sum_{i, j}^{d_{\pi^{\alpha}}}\left|\hat{g}\left(\pi^{\alpha}\right)_{i, j}\right|^{2} \leq \int_{K}|g(x)|^{2} d m(x) \leq N_{\Psi}\left(g^{2}\right),
$$

where the last inequality follows from Hölder's inequality (since $N_{\Phi}(1)=1$ ). Set $\Psi_{1}(t)=\Psi\left(t^{2}\right)$. Then $\Psi_{1}$ is a Young function satisfying $\Psi \prec \Psi_{1}$. Since $g$ is a bounded function, by setting $a^{2}=N_{\Psi}\left(g^{2}\right)(<\infty)$, we get

$$
\Psi_{1}(1)=\Psi(1)=\int_{K} \Psi\left(\frac{|g|^{2}}{a^{2}}\right) d m(x)=\int_{K} \Psi_{1}\left(\frac{|g|}{a}\right) d m(x),
$$

whence $a=N_{\Psi_{1}}(g)$. Thus, by (3.18), we have

$$
S_{2}\left(\tilde{g}_{\Lambda_{0}}\right) \leq N_{\Psi_{1}}(g) .
$$

Now, we find an absolute bound for $M$. If $a=N_{\Psi_{1}}(g)$ then, by definition, there exists $b_{0}>0$ such that

$$
1=\int_{K} \Psi_{1}\left(\frac{g}{a b_{0}}\right) d m(x)=\int_{K} \Psi_{1}\left[\frac{1}{a b_{0}} \Psi^{\prime}\left(\frac{\left|f_{\Lambda_{0}}\right|}{N_{\Psi}\left(f_{\Lambda_{0}}\right)}\right)\right] d m(x) .
$$

Since $\Psi^{\prime}(t) \leq c_{0} t^{p}$ for some $p \geq 1$, we get

$$
1 \leq \int_{K} \Psi_{1}\left[\frac{c_{0}}{b_{0} a}\left(\frac{\left|f_{\Lambda_{0}}\right|}{N_{\Psi}\left(f_{\Lambda_{0}}\right)}\right)^{p}\right] d m(x)=\int_{K} \Psi_{2}\left[b_{1} \frac{\left|f_{\Lambda_{0}}\right|}{N_{\Psi}\left(f_{\Lambda_{0}}\right)}\right] d m(x)
$$

where $\Psi_{2}(t)=\Psi_{1}\left(t^{p}\right)$ and $b_{1}=\left(\frac{c_{0}}{b_{0} a}\right)^{\frac{1}{p}}>0$. Thus $\Psi_{2}$ is a Young function satisfying $\Psi \prec \Psi_{1} \prec \Psi_{2}$. Then (3.20) gives the following important inequality: there exists a constant $b_{2}$ depending only on $\Psi_{2}$ and independent of $f_{\Lambda_{0}}$, such that

$$
N_{\Psi_{2}}\left(\frac{b_{1} f_{\Lambda_{0}}}{N_{\Psi}\left(f_{\Lambda_{0}}\right)}\right) \geq b_{2}>0
$$

(see [11, Theorem 2, Chapter III]). Since $N_{\Psi_{2}}(\cdot)$ is a norm, by the definition of $b_{1}$, we get

$$
\left[\frac{N_{\Psi_{2}}\left(f_{\Lambda_{0}}\right)}{N_{\Psi}\left(f_{\Lambda_{0}}\right)}\right]^{p} \geq b_{2}^{p}\left(\frac{b_{0}}{c_{0}}\right) N_{\Psi_{1}}(g)=b_{3} N_{\Psi_{1}}(g),
$$

where $b_{3}=b_{2}^{p} \frac{b_{0}}{c_{0}}$. Since we have $\Psi_{0} \prec \Psi \prec \Psi_{1} \prec \Psi_{2}$, by Lemma 2.1, $\Phi_{2} \prec \Phi_{1} \prec \Phi \prec \Phi_{0}$ so that $\ell^{\Phi_{2}} \subset \ell^{\Phi_{1}} \subset \ell^{\Phi} \subset \ell^{\Phi_{0}}=\ell^{2} \subset \ell^{\Psi} \subset \ell^{\Psi_{1}} \subset \ell^{\Psi_{2}}$. Now, for some $r_{2}>0$, we get the following inequalities:

$1 \unlhd 3 \mathrm{D} 23 \neq M_{\Phi}=S_{\Psi}\left(\tilde{g}_{\Lambda_{0}}\right) \leq r_{2} S_{2}\left(\tilde{g}_{\Lambda_{0}}\right) \leq \frac{r_{2}}{b_{3}}\left[\frac{N_{\Psi_{2}}\left(f_{\Lambda_{0}}\right)}{N_{\Psi}\left(f_{\Lambda_{0}}\right)}\right]^{p} \leq \frac{r_{2}}{b_{3}}\left[\frac{N_{\Psi_{3}}\left(f_{\Lambda_{0}}\right)}{N_{\Psi}\left(f_{\Lambda_{0}}\right)}\right]^{p}$, 
where $\Psi_{3}(t)=\frac{c_{0}}{p+1}|t|^{2 p(p+1)}$, so $\Psi_{2} \prec \Psi_{3}$, (Since $\Psi^{\prime}(t) \leq c_{0} t^{p}$ ). Let $\Phi_{3}$ be the complementary function of $\Psi_{3}$. Then $S_{\Phi_{3}}\left(f_{\Lambda_{0}}\right) \leq b_{4} S_{\Phi}\left(f_{\Lambda_{0}}\right)$ for some $b_{4}>0$ depending only on $\Phi_{3}$ and $\Phi$ only. Therefore, by (3.23), we have

$$
1 \leq M=M_{\Phi} \leq \frac{r_{2}}{b_{3}}\left[\frac{M_{\Phi_{3}} S_{\Phi_{3}}\left(f_{\Lambda_{0}}\right)}{M_{\Phi} S_{\Phi}\left(f_{\Lambda_{0}}\right)}\right]^{p} \leq \frac{r_{2}}{b_{3}}\left[b_{4} \frac{M_{\Phi_{3}}}{M_{\Phi}}\right]^{p}
$$

Hence,

$$
1 \leq M_{\Phi}^{p+1} \leq r_{3}^{p} M_{\Phi_{3}}^{p}
$$

for some positive constant $r_{3}>0$ which depend only on $\Phi, \Phi_{2}, \Phi_{3}$ and the ordering constants. But note that $L^{\Psi_{3}}(K)=L^{p^{\prime}}(K)$, where $p^{\prime}=2 p(p+1) \geq$ 2. It follows from Theorem 3.1 that $M_{\Phi_{3}} \leq r_{4}<\infty$, for a positive constant $r_{4}$ depending on $c_{0}$ and $r$. Therefore (3.25) gives

$$
1 \leq M_{\Phi} \leq r_{5}<\infty
$$

where $r_{5}=\left(r_{3} r_{4}\right)^{\frac{p}{p+1}}$, which is independent of $\Lambda_{0}$.

STEP III. By setting $\bar{r}_{0}=r_{5}$, equations (3.9) immediately give the required inequality in (3.8). Since $\Psi_{2}$ and $\Psi_{3}$ depend on the complementary Young function $\Psi$ of $\Phi$, all the constants involve depend on $\Phi$ and the ordering $\Phi \prec$ $\Phi_{0}$, and perhaps on $c_{0}$ and $p$. This completes the proof of the lemma.

Now, we are ready to prove the Hausdorff-Young inequality for Orlicz spaces on compact hypergroups.

Main Theorem 3.3. Let $K$ be a compact hypergroup with the normalized Haar measure $m$ and let $(\Phi, \Psi)$ be a pair of continuous normalized Young functions such that

(i) $\Phi \prec \Phi_{0}$, where $\Phi_{0}(t)=\frac{1}{2}|t|^{2}$,

(ii) $\Psi^{\prime}(t) \leq c_{0} t^{p}, t \geq 0$, for some $p \geq 1$,

where $c_{0}$ is a positive constant. If $f \in M^{\Phi}(K)$ then there is $r_{0} \geq 1$ such that

$$
N_{\Psi}\left(F_{f}\right) \leq r_{0} N_{\Phi}(f) .
$$

Proof. Let $f \in M^{\Phi}(K)$ and let $\Lambda_{0}$ be finite subset of $\Lambda$. Suppose $\tilde{f}_{\Lambda_{0}}$ is given by (3.7) where $c_{i, j}^{\alpha}=\hat{f}\left(\pi_{i, j}^{\alpha}\right)$. It is known that the set $\left\{\tilde{f}_{\Lambda_{0}}: \Lambda_{0} \subset \Lambda\right\}$ of such functions is dense in $M^{\Phi}(K)$ (see [16] and [7]). If $\left\{F_{\tilde{f}_{\Lambda_{0}}}(\alpha): \alpha \in \Lambda_{0}\right\}$ is corresponding function in $\ell^{\Psi}\left(k^{2}\right)$, then we have

$$
\lim _{\Lambda_{0} \subset \Lambda} N_{\Phi}\left(f-\tilde{f}_{\Lambda_{0}}\right)=0, \text { and } \lim _{\Lambda_{0} \subset \Lambda} N_{\Psi}\left(F_{\tilde{f}_{\Lambda_{0}}}\right)=N_{\Psi}\left(F_{f}\right),
$$

where the limit as $\Lambda_{0}$ varies in $\Lambda$ is taken using the partial order define by inclusion of subset of $\Lambda$. Now, by using this with the inequality (3.8), $N_{\Psi}\left(F_{f_{\Lambda_{0}}}\right) \leq \bar{r}_{0} N_{\Phi}\left(f_{\Lambda_{0}}\right)$, of Lemma 3.2 , we get $N_{\Psi}\left(F_{f}\right) \leq r_{0} N_{\Phi}(f)$, where $r_{0}=\bar{r}_{0}$. This completes the proof. 
Remark 3.4. (i) Examples of Young functions $\Phi$ satisfying the conditions in Theorem 3.3 but different from $|x|^{p} / p$ can be found in $[12$, p. 226].

(ii) For the Lebesgue space, constant $r_{0}$ is simply 1 (see Theorem 3.1); however it is clear from the proof of Lemma 3.2 that the constant $r_{0}$ in Theorem 3.3 is greater or equal to 1 .

Remark 3.5. It would be interesting to find whether the Hausdorff-Young inequlity for Orlicz spaces holds if $K$ is a commutative hypergroup. For a locally compact abelian group, this inequality was established by M. M. Rao [8]. An obvious generalization of the techniques does not work as Rao uses the structure theorem of locally compact abelian groups.

Acknowledgements. The authors thank the anonymous referees for their kind suggestions. The first author thanks the Council of Scientific and Industrial Research, India, for its research grant.

\section{REFERENCES}

[1] W. R. Bloom and Herbert Heyer, Harmonic analysis on probability measures on hypergroups, De Gruyter, Berlin (1995) (Reprint: 2011).

[2] A. P. Caldron and A. Zygmund, A note on the interpolation of sublinear operator, Amer. J. Math., 78(2) (1956), 282-288.

[3] S. Degenfeld-Schonburg, On the Hausdorff-Young theorem for commutative hypergroups, Colloq. Math., 131(2) (2013) 219-231.

[4] C. F. Dunkl, The measure algebra of a locally compact hypergroup, Trans. Amer. Math. Soc., 179 (1973) 331-348.

[5] G. H. Hardy and J. E. Littlewood, Some new properties of Fourier constant, Math. Annalen 97 (1927) 159-209.

[6] R. I. Jewett, Spaces with an abstract convolution of measures, Adv. Math., 18 (1975) 1-101.

[7] V. Kumar, R. Sarma and N. S. Kumar, Orlicz spaces on hypergroups, Publ. Math. Debrecen, 94(1-2) (2019) 31-47.

[8] M.M. Rao, Extension of the Hausdorff-Young Theorem, Israel J. Math., 6 (1967) 133-149.

[9] M. M. Rao, Convolution of vector fields-II : random walk models, Nonlinear Analysis, 47 (2001) 3599-3615.

[10] M. M. Rao, Convolutions of vector fields-III: Amenability and spectral properties, Real and stochastic analysis, Trends Math., Birkhäuser Boston, Boston, MA, (2004) 375-401. 
[11] M.M. Rao and Z.D. Ren, Theory of Orlicz spaces, Dekker, New York (1991).

[12] M. M. Rao and Z. D. Ren, Applications of Orlicz spaces Monographs and Textbooks in Pure and Applied Mathematics, 250, Marcel Dekker Inc., New York, (2002).

[13] K. A. Ross, Centers of hypergroups, Trans. Amer. Math. Soc., 243 (1978) 251-269.

[14] K. A. Ross, Hypergroups and centers of measure algebras, Symposia Mathematica, Vol. XXII (Convegno sull'Analisi Armonica e Spazi di Funzioni su Gruppi Localmente Compatti, INDAM, Rome, 1976), Academic Press, London, (1977) 189-203.

[15] R. C. Vrem, Representations and Harmonic analysis of compact hypergroups, Doctoral Dissertation, University of Oregon (1978).

[16] R. C. Vrem, Harmonic analysis on compact hypergroups, Pacific $J$. Math., 85(1) (1979) 239-251.

[17] A. Zygmund, Trigonometry series Vol. I and II combined, third edition, with a foreword by Robert A. Fefferman, Cambridge mathematical library, Cambridge University Press, Cambridge, (2002).

Department of Mathematics, Indian Institute of Technology Delhi, Delhi - 110 016, India.

Email address: vishveshmishra@gmail.com

Department of Mathematics, Indian Institute of Technology Delhi, Delhi - 110 016, InDIA.

Email address: ritumoni@maths.iitd.ac.in 